\title{
Effective Symmetry Breaking of Flow in AC Electro-Osmotic Pump Using a Ratchet
}

\section{Structure}

Hideyuki Sugioka ${ }^{1, a)}$ and Satoru Segawa ${ }^{1}$

${ }^{1}$ Department of Mechanical Systems Engineering, Shinshu University 4-17-1

Wakasato, Nagano 380-8553, Japan

(Dated: 13 June 2019)

AC electro-osmotic (ACEO) pumps play an important role in a wide diversity of microfluidic applications. To produce high flow velocities, an effective breaking of symmetry is prerequisite. Here, we introduced a ratchet-structured electrode for achieving high flow velocities and developed a ratchet ACEO pump containing both ratchet and plane electrodes. The performance of our new ACEO pump is evaluated experimentally. At an ac voltage of $30 \mathrm{~V}$ at $10 \mathrm{kHz}$, a maximum net flow velocity of $1.1 \mathrm{~mm} / \mathrm{s}$ is measured along the inclined direction of the ratchet teeth. Furthermore, we also directly observed the flow fields due to the slip velocity of ACEO, which provide a physical insight on the ratchet-type ACEO pumps.

PACS numbers: 47.57.-s, 85.90.+h, 82.45.Hk, 83.50.Lh

a)Electronic mail: hsugioka@shinshu-u.ac.jp 


\section{INTRODUCTION}

Pumps play a central role in a wide diversity of microfluidics applications such as lab-ona-chip and micro total analysis systems ${ }^{1}$. Thus, a diverse variety of pumps from a mechanical type to a non-mechanical type have been investigated so far ${ }^{2}$. In particular, pumps that use ac electro-osmosis (ACEO) or induced charge electro-osmosis (ICEO) are attractive as a non-mechanical pump ${ }^{3-5}$. This is because they produce large flow velocities $(\sim 1 \mathrm{~mm} / \mathrm{s})$ at low applied voltages $(\sim 1 \mathrm{~V})$ compared to ordinary electro-osmotic pumps, which only reach a velocity of $0.1 \mathrm{~mm} / \mathrm{s}$ even at a high voltage of $1 \mathrm{kV}^{6}$. The $\mathrm{ACEO}^{7}$ and the advanced concept, $\mathrm{ICEO}^{8}$, are the phenomena that occur in the polarizable materials and are characterized by the flow velocity proportional to the square of the applied electric field. The ACEO pumps using asymmetric arrays of electrodes were first proposed by Ajdari theoretically ${ }^{3}$ and developed to the most promising electro-osmotic pumps by the efforts of several groups ${ }^{4,5,9}$. However, the high flow velocity $(\sim 1 \mathrm{~mm} / \mathrm{s})$ of the array-type ACEO pump is a consequence of optimum design efforts ${ }^{5,10}$. Specifically, on the basis of the theoretical design introduced by Bazant and Ben ${ }^{10}$, Urbanski et al. ${ }^{5}$ experimentally demonstrated that the ACEO pump with three-dimensional (3D) electrode arrays, consisting of asymmetrically placed step electrodes in a symmetric planar array, can reach a higher velocity of $\sim 0.4 \mathrm{~mm} / \mathrm{s}$. Although this design is quite elegant, it produces an unwanted reverse vortex flow, which is observed to be very sensitive to the dimensions of the step electrode.

This unwanted reverse vortex flow results from the array electrode structure placed in the flow direction on the substrate (e.g., see Fig. 1 in Ref. ${ }^{5}$ ) and, thus, they exist intrinsically. Thus, the optimization of the dimensions of the step electrodes is essential for suppressing the reverse vortex flow. In our pump design, we changed the electrode configuration to suppress the unwanted local flows. Further, although the 3D array-type ACEO pump is attractive, it only has a local asymmetrical structure that produces a local asymmetrical ACEO flow. In other words, it does not have a global asymmetrical structure, such as that in the ratchet structure ${ }^{11,12}$. Note that the ratchet structure can provide an asymmetrical flow resistance of the whole channel. Indeed, Groisman and Quake ${ }^{11}$ have reported that a microfluidic channel with a ratchet structure works as a microfluidic rectifier for the nonNewtonian fluids. Linke ${ }^{12}$ also reported that a water droplet above a hot ratchet moves along the inclined direction of the ratchet tooth resulting from the asymmetrical flow of the 
vapor layer due to the Leidenfrost phenomenon, which is a type of film boiling phenomenon. However, to the best of our knowledge, no any ACEO pump using the ratchet structure is reported. Here, the asymmetrical flow is ideally a flow in one direction without reverse flow, whereas the net flow of the array-type ACEO pump generally consists of local forward and reverse flows having different flow velocities. Thus, a net flow is reduced by the existence of the reverse flow.

Therefore, we consider using a ratchet-type electrode with a configuration that sandwiches a liquid between the plane electrode and the ratchet-type electrode to obtain not only a local asymmetrical ACEO flow but also an asymmetrical flow resistance over the whole channel. In this paper, we report a novel ratchet ACEO pump and the performance of this pump is evaluated experimentally. of $\sim 1 \mathrm{~mm} / \mathrm{s}$ of an electrokinetic pump ${ }^{5}$. Furthermore, by the direct observation of the channel, we show that this net flow is obtained without producing any unwanted vortex flow in the steady state.

\section{METHOD}

Figure 1 shows a schematic view of the experimental setup of the ACEO pump using a ratchet-type electrode. As shown in Fig. 1, the ratchet-type ACEO pump comprises a sandwich structure with an Au-coated aluminum ratchet-type electrode of length $L(=20$ $\mathrm{mm})$ and depth $D(=20 \mathrm{~mm})$, a water channel, and an Au-coated copper plane electrode. The $\mathrm{Al}$ ratchet-type electrode and the $\mathrm{Cu}$ electrode were coated by an $\mathrm{Au}$ film of thickness $30 \mathrm{~nm}$ to suppress the chemical reactions at the electrodes; the distance $W$ between the electrodes was $1.5 \mathrm{~mm}$. Further, the ratchet pump was immersed in a water pool of length $L^{\prime}=75 \mathrm{~mm}$, width $W^{\prime}=22 \mathrm{~mm}$, and depth $D^{\prime}=25 \mathrm{~mm}$. By applying an alternate square wave voltage of frequency $f_{0}$ (typically, $100 \mathrm{~Hz}$ ) between the electrodes, we observed a strong ACEO flow ${ }^{7}$ of the order $\sim 1 \mathrm{~mm} / \mathrm{s}$ for our ratchet pump. The mechanism is simply illustrated in Fig. 1. When we apply a voltage of $V_{0}$ (typically, $15 \leq V_{0} \leq 35 \mathrm{~V}$ ), an induced charge is produced around the peak of the ratchet. As this induced charge attracts the counter ion, the electric double layer of the zeta potential $\zeta\left(\sim 0.5 V_{0}\right)$ is formed around the ratchet peak. The charge in the electric double layer interacts with the tangential electric field $E_{t}\left(\sim V_{0} / W\right)$, and hence, the fluid in the electric double layer moves. By substituting $\zeta \sim V_{0} / 2$ and $E_{t} \sim V_{0} / W$ in the Smoluchowski formula $V_{s}=\frac{\varepsilon \zeta E_{t}}{\mu}$ with a correction factor of 


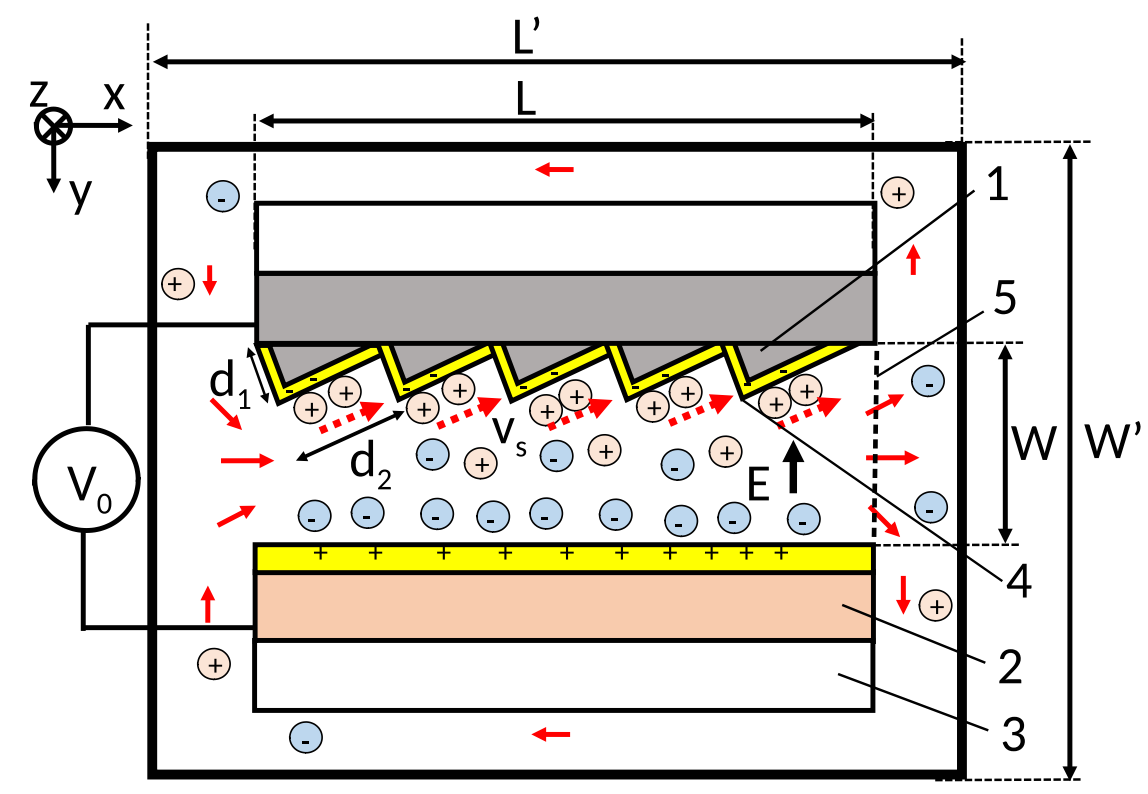

FIG. 1. (Color online) Schematic view of the experimental setup of the ratchet ACEO pump. Here, the depth of water $D^{\prime}$ is $25 \mathrm{~mm}$, whereas the depth of the ratchet $D$ is $20 \mathrm{~mm} ; W^{\prime}=22 \mathrm{~mm}$, $L^{\prime}=75 \mathrm{~mm}, W=1.5 \mathrm{~mm}, L=20 \mathrm{~mm}, d_{1}=0.3 \mathrm{~mm}$, and $d_{2}=1.2 \mathrm{~mm} .1$ : Au-coated Al ratchet, 2: Au-coated $\mathrm{Cu}$ electrode, 3: slide glass, 4: summit of the ratchet, 5: outlet of the channel.

$\Lambda^{8,13}$, which is originally introduced to consider the Stern layer, we obtain the slip velocity $V_{s} \sim \frac{\varepsilon V_{0}^{2}}{2 \mu W} \Lambda$. This slip velocity $V_{s} \sim \frac{\varepsilon V_{0}^{2}}{2 \mu W} \Lambda$ is generated at the outer edge of the electric double layer, where $\varepsilon \simeq 80 \varepsilon_{0}$ is the dielectric permittivity, $\varepsilon_{0}$ is the vacuum permittivity, and $\mu=1 \mathrm{mPa} \mathrm{s}$ is the viscosity of water, which is originally introduced for the Stern layer. Note that $D$ and $D^{\prime}$ are the quantities in the $z$ direction in Fig. 1; i.e., the direction of the gravity is in the $z$ direction (i.e., the vertical direction to the sheet).

\section{RESULTS}

Figure 2 shows the time evolution of the flow fields of the ratchet-type ACEO pump under the condition that $V_{0}=25 \mathrm{~V}$ and $f_{0}=10 \mathrm{kHz}$. In Fig. $2, f_{a}$ is the relative magnification factor of the flow vector. As shown in Fig. 2(a), in the early stage at $t=3.3 \mathrm{~s}$, we observed plural vortex flows whose size approximately corresponds to the period of the ratchet (i.e., 


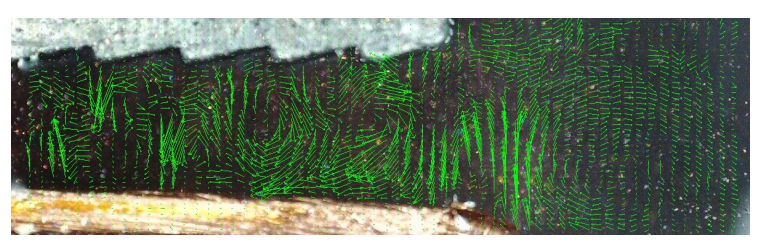

(a) $t=3.3 \mathrm{~s}\left(f_{a}=2.5\right)$

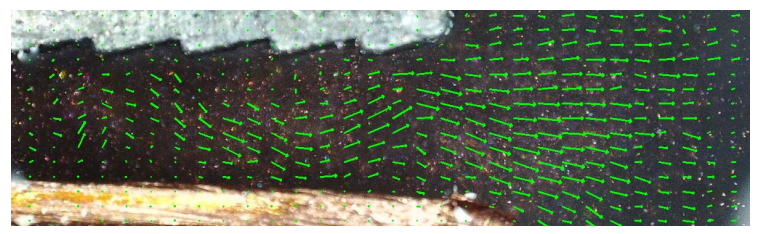

(c) $t=20.2 \mathrm{~s}\left(f_{a}=1.0\right)$

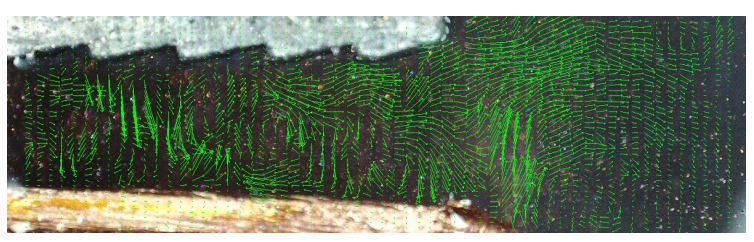

(b) $t=5.3 \mathrm{~s}\left(f_{a}=2.5\right)$

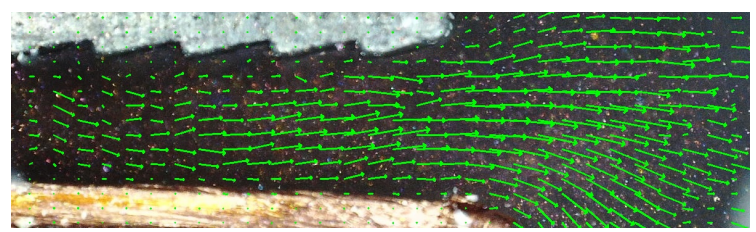

(d) $t=44.0 \mathrm{~s}\left(f_{a}=1.0\right)$

FIG. 2. (Color online) Time evolution of the flow fields of the ratchet ACEO pump. Here, $V_{0}=25$ $\mathrm{V}, f_{0}=10 \mathrm{kHz}$, and $W=1.5 \mathrm{~mm} ; f_{a}$ is the relative magnification factor of the flow vector.

$\sqrt{d_{1}^{2}+d_{2}^{2}} \simeq 1.24 \mathrm{~mm}$ ). This is because the slip velocity $V_{s}$ along the gentle slope causes each vortex flow initially, because of the fluidic inertance of the fluidic system. Note that to accelerate a fluid, a force is required, and the fluidic inertance originates from the inertial mass of the fluid. Then, we observed that the vortex flows gradually disappeared from the vicinity of the outlet, as shown in Figs. 2(b) and 2(c). This process is also explained by the fact that the partial forward fluidic inertance $I_{f}=x_{f} \rho / A_{c}$ is small near the vicinity of the outlet, where $\rho\left(\sim 1000 \mathrm{~kg} / \mathrm{m}^{3}\right)$ is the density of water, $A_{c}(\simeq W D)$ is the crosssectional area of the channel, and $x_{f}$ is the distance between the considered position and the outlet position. Here, we assume that $d_{2} \ll W$, i.e., we neglect the ratchet structure for the inertance for simplicity. Note that the partial forward and rear fluidic inertances are the inertances in the forward and rear regions, respectively of the channel consisting of the ratchet and plane electrodes ${ }^{14}$. Further, at the same time, we observed that the size of the vortex becomes large as the vortex flows disappear, as shown in Figs. 2(a) to 2(c). Finally, at $t=44 \mathrm{~s}$, we observed a steady net flow in the direction of $x$ without vortex flows, as shown in Fig. 2(d).

Figure 3 shows the characteristics of the ratchet ACEO pump. Specifically, Fig. 3(a) shows the dependence of the velocity $v_{x}$ in the $x$ direction on $y$ at $t=6.13,29.47$, and $116.13 \mathrm{~s}$ at the outlet position. As shown in Fig. 3(a), we found that the distributions of $v_{x}$ in the ratchet ACEO pump are similar to those of Poiseuille flows (at least at $t>6 \mathrm{~s}$ ), 


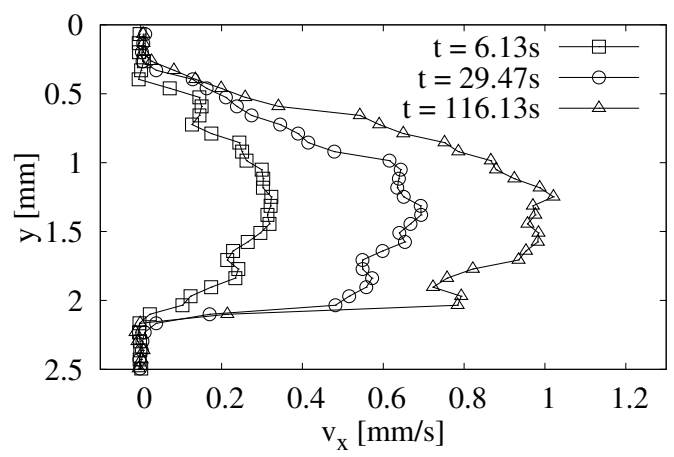

(a) Dependence of $v_{x}$ on $y\left(f_{0}=10 \mathrm{kHz}, V_{0}=30\right.$

V)

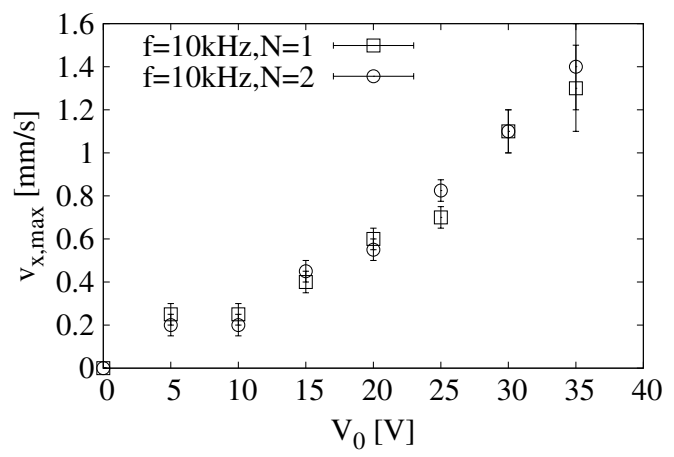

(c) Dependence of $v_{x, \max }$ on $V_{0}\left(f_{0}=10 \mathrm{kHz}\right)$

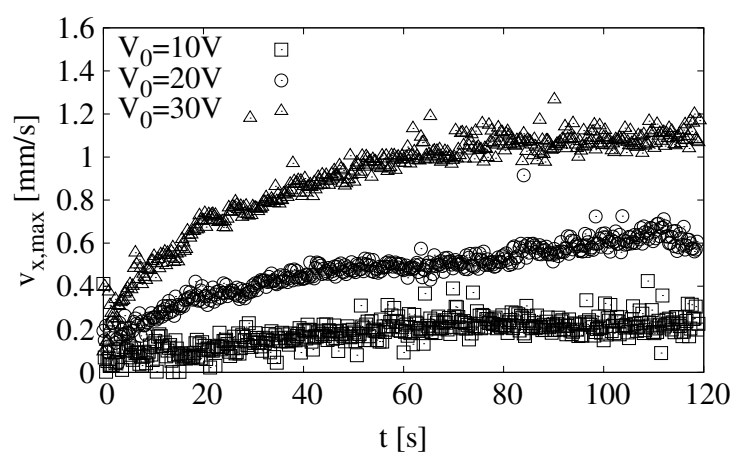

(b) Dependence of $v_{x, \max }$ on $t\left(f_{0}=10 \mathrm{kHz}\right)$

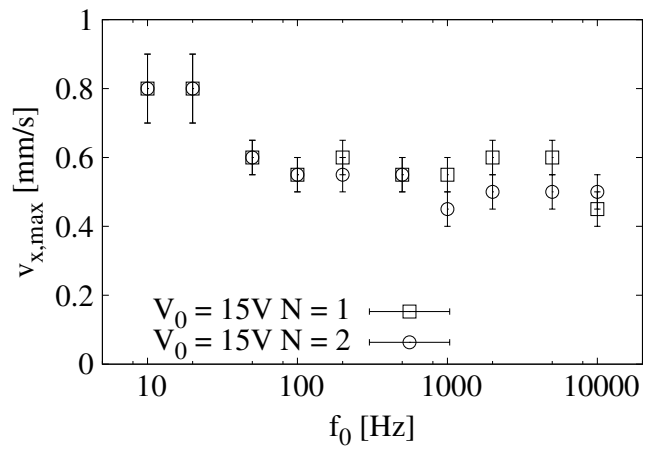

(d) Dependence of $v_{x}^{\text {steady }}$ on $f_{0}\left(V_{0}=15 \mathrm{~V}\right)$

FIG. 3. Characteristics of the ratchet ACEO pump. Here, $W=1.5 \mathrm{~mm}$.

although we assume the slip velocity on the slope of the ratchet electrode. This is because the strong slip velocity only exists around the peak of the ratchet and, thus, a viscus flow becomes dominant in this system. Fig. 3(b) shows the dependence of the maximum flow velocity $v_{x, \max }$ on $t$ at $V_{0}=10,20$, and $30 \mathrm{~V}$ at $f_{0}=10 \mathrm{kHz}$, where $v_{x, \text { max }}$ is the maximum value of $v_{x}$ in the region that $0 \leq x_{f} \leq 1.5 \mathrm{~mm}$. As shown in Fig. 3(b), $v_{x \text {,max }}$ increases gradually and becomes a steady state at $t \simeq 100 \mathrm{~s}$. That is, $v_{x, \max }$ is $0.2,0.6$, and 1.1 $\mathrm{mm} / \mathrm{s}$, respectively at the steady state at $V_{0}=10,20$, and $30 \mathrm{~V}$. Figure $3(\mathrm{c})$ shows the dependence of $v_{x, \max }$ at the steady state on $V_{0}$ at $f_{0}=10 \mathrm{kHz}$. As shown in Fig. 3(c), we found that our ratchet pump has a nonlinear voltage characteristic described by $v_{x} \propto V_{0}^{2}$. Thus, we consider that our ratchet pump is driven by the ACEO flow with a high maximum flow velocity as an electro-osmotic pump (e.g., $U_{\max }=v_{x, \max } \sim 1.4 \mathrm{~mm} / \mathrm{s}$ at $V_{0}=35 \mathrm{~V}$ and 
$W=1.5 \mathrm{~mm})$. Figure $3(\mathrm{~d})$ shows the dependence of $v_{x}$ at the steady state on $f_{0}$ at $V_{0}=15$ V. As shown in Fig. 3(d), we found that the ratchet ACEO pump works on a wide frequency range, although $v_{x}$ gradually decreases from 0.8 to $0.5 \mathrm{~mm} / \mathrm{s}$ at $V_{0}=15 \mathrm{~V}$ in the range $10 \leq f_{0} \leq 10000 \mathrm{~Hz}$. This is because there is no unwanted vortex flow in the ratchet ACEO pump at the steady state and, thus, the flow fields are not sensitive to $f_{0}$. In other words, a ratchet $\mathrm{ACEO}$ pump outperforms a 3D array-type ACEO pump in terms of frequency characteristic. Note that we could not perform our pumping experiment in the range less than $10 \mathrm{~Hz}$ because of the generation of bubbles due to the electrolysis at the electrodes, although our pump works theoretically even in the range less than $10 \mathrm{~Hz}$.

\section{DISCUSSION}

\section{A. Main Features of Our Ratchet-Type ACEO Pump}

Although the 3D array-type ACEO pump has already been reported ${ }^{5,10}$, here, we demonstrated the performance of our ACEO pump using the ratchet structure. Thus, by using a sandwich structure consisting of the ratchet-type electrode and the plane electrode, we succeeded in generating a natural net directional flow due to the ACEO slip velocity along to the gentle slope of the ratchet electrode in the presence of the electric field perpendicular to the channel direction. Further, by this new structure, we succeeded in observing a high net flow velocity (e.g., $U_{\max } \sim 1.4 \mathrm{~mm} / \mathrm{s}$ ) without any complicated optimum design. In other words, the ratchet ACEO pump enables robust designs because it does not produce any unwanted vortex flow. In fact, $U_{\max }\left(=v_{x, \max }\right)$ is insensitive to $f_{0}$ [see Fig. $3(\mathrm{~d})$ ] and, thus, the working frequency range of the ratchet ACEO pump is preferable. This is very different from the 3D array-type ACEO pump that has typically two large peaks in frequency characteristic ${ }^{5}$. In addition, by using a ratchet ACEO pump connected to a water pool having large inertance, we succeeded in observing both the vortex flow due to an ACEO slip velocity in the initial state and the natural parabolic net directional flow without an unwanted vortex flow in the steady state [in Fig. 2] using the same system. This is the direct evidence of the physical mechanism and the proof-of-concept of the ratchet ACEO pump. 


\section{B. Flow State and Fluidic Circuit Model}

Here, we discuss a flow state and fluidic circuit model for our experiment to understand the performance of our pump. If we assume a parabolic flow (based on the result of Fig. 3(a)) for the steady state, the average flow velocity $U_{\text {ave }}$ is obtained as $U_{\text {ave }}=\frac{2}{3} U_{\max } \simeq 0.94 \mathrm{~mm} / \mathrm{s}$ for $U_{\max } \simeq 1.4 \mathrm{~mm} / \mathrm{s}$. Moreover, the steady volume flow rate $q_{0}$ is obtained as $q_{0}=A U_{\text {ave }} \simeq 28$ $\mathrm{mm}^{3} / \mathrm{s}$. Further, if we assume that the system is described by the simple fluidic circuit model $^{14,15}$ of the total fluidic inertance $I_{t}\left(=I_{c}+I_{\mathrm{ex}}\right)$ and resistance $R_{t}\left(=R_{c}+R_{\mathrm{ex}}\right)$ as the first step, the governing equation of the system is provided as $\Delta P=R_{t} q+I_{t} \frac{d q}{d t}$ and the solutions are provided as $q=\frac{\Delta P}{R}\left(1-\mathrm{e}^{-\frac{t}{\tau}}\right)$, where $q$ is the volume flow rate, $\Delta P$ is the generated pressure, $\tau=\frac{I_{t}}{R_{t}}$ is the time constant of the step response, and $I_{c}$ and $I_{\mathrm{ex}}\left(R_{c}\right.$ and $R_{\mathrm{ex}}$ ) are the inertances (resistances) of the channel and external regions, respectively. Thus, we can improve $\tau$ [experimentally, $\sim 30$ s from Fig. 3(b)] in the future by reducing $I_{t}$. Here, the fluidic circuit model ${ }^{14,15}$ is a model using an analogy between electric and fluid circuits and it is often used to analyze complex flow systems such as a flow system in an ink jet printer ${ }^{16}$. Specifically, from the Hagen-Poiseuille law, we obtain $\Delta P=\frac{12 \mu}{W^{3}} \frac{L}{D} q$, i.e., $R_{c}=\frac{12 \mu}{W^{3}} \frac{L}{D}=3.55 \times 10^{6} \mathrm{~kg} / \mathrm{m}^{4} \mathrm{~s}$ for our pump. Since we can neglect the flow resistance of the wide external region (i.e., $R_{e x} \ll R_{c}$ ), we obtain $R_{t} \simeq R_{c}$. Further, from Newton's second law, we obtain $f=A_{c} \Delta P=m_{t} \frac{d U_{a v e}}{d t}=m_{t} \frac{d q}{A_{c} d t}$, where $m_{t} \simeq D^{\prime} W^{\prime} L^{\prime} \rho$ is the total mass of water in the pool and $A_{c}=D W$ is a cross-sectional area of the channel. Thus, we obtain $\Delta P \simeq \frac{m_{t}}{A_{c}^{2}} \frac{d q}{d t}$, i.e., $I_{t} \simeq \frac{m_{t}}{A_{c}^{2}} \simeq 4.6 \times 10^{7} \mathrm{~kg} / \mathrm{m}^{4}$ for our device. However, the prediction of $\tau=I_{t} / R_{f} \sim 13 \mathrm{~s}$ is much smaller than the experimental result $(\sim 30 \mathrm{~s})$. Therefore, we consider that the flow model should be improved in the future. In particular, $\Delta P\left(=q_{0} R_{t} \simeq 0.1 \mathrm{~Pa}\right)$ may be considered to be an apparent generated pressure and it should be replaced by $\Delta P^{\prime}-\Delta P_{\mathrm{ex}}$, where $\Delta P^{\prime}$ is a true generated pressure and $\Delta P_{\mathrm{ex}}$ is an external pressure generated by the elevation of water surface. However, this is a complex nonlinear phenomenon and it is the beyond the scope of this manuscript.

\section{High Maximum Flow Velocity as an Electro-Osmotic Pump}

Usually, the flow velocity depends on various conditions such as the device size, external circuit, and so on. For example, the maximum generated pressure $\Delta P_{\max }$ of a recipro- 
cating displacement micropump (a kind of diaphragm pump) is theoretically described as $\Delta P_{\max }^{\text {diaphragm }}=\frac{1}{\kappa}\left(\frac{\Delta V}{V_{\text {dead }}}\right)$, where $\Delta V$ is the stroke volume, $V_{\text {dead }}$ is the dead volume, and $\kappa$ is the compressibility of the working fluid; and the increase of $\Delta P_{\max }$ increases the maximum volume flow rate and the flow velocity ${ }^{17}$. However, for the ACEO pump, the intrinsic flow velocity is determined by the electro-kinetic slip velocity $U_{\text {slip }}$ at the outer edge of the electric double layer and the efficiency of the broken symmetry of $U_{\text {slip }}$. Thus, the maximum net flow velocity $U_{\text {ave }}^{\max }$ over the electrode (or device region) is the most important index of the performance although the resistance of the external circuit $R_{\mathrm{ex}}$ affects the flow velocity as $U_{\text {ave }} \simeq \Delta P /\left(R_{c}+R_{\mathrm{ex}}\right) A$ as discussed in Sec. IV-B. In this context, as a state of the art, Urbanski et al. showed $U_{\text {ave }}^{\max }=0.42 \mathrm{~mm} / \mathrm{s}$ (at $V_{0}=1.06 \mathrm{~V}_{\mathrm{RMS}}$ ) by using the 3D array-type ACEO pump, in their first report ${ }^{5}$ and Huang et al. showed $U_{\text {ave }}^{\max }=1.3 \mathrm{~mm} / \mathrm{s}$ (at $V_{0}=1.06$ $\mathrm{V}_{\mathrm{RMS}}$ ) by using an optimized one later with the argument of a generated pressure ${ }^{18}$. Thus, in this sense, our ratchet pump is driven by the ACEO flow with a high maximum flow velocity $U_{\text {ave }}^{\max }=0.94 \mathrm{~mm} / \mathrm{s}$. Note that from the above argument, $I_{\mathrm{ex}}$ decreases and $R_{\mathrm{ex}}$ increases, as the pool size becomes small. Thus, $\tau$ and $q$ (or $U_{\text {ave }}$ ) decrease as the pool size becomes small. In particular, when the gap of the external channel (i.e, the gap between the frame of the pool and the device) becomes compatible with $W$, the effect of $R_{\text {ex }}$ cannot be negligible.

\section{Maximum Volume Flow Rate per Unit Width}

Although it has not been discussed well so far, the maximum volume flow rate per unit width over the electrode (or device region) (i.e., $U_{\text {ave }}^{\max } D$ ) is also an important parameter to determine the performance of ACEO pumps. Since $D=25 \mu \mathrm{m}$ and $U_{\text {ave }}^{\max }=1.3 \mathrm{~mm} / \mathrm{s}$ for the 3D array-type ACEO pump of Huang et al. ${ }^{18}$, the ability to convey a liquid of the interfacial flow of our ratchet ACEO pump is approximately $43(\simeq 1500 \times 0.94 / 25 \times 1.3)$ times larger than that of the 3D array-type ACEO pump. Note that this ability is important for the cooling of microelectronic devices since microelectronics cooling is highly demanding with respect to flow rate, e.g., a flow as high as several hundred milliliters per minute is often required for this purpose ${ }^{17}$. In this sense, the performances of $q_{0}=28 \mathrm{~mm}^{3} / \mathrm{s}(=$ $1680 \mathrm{ml} / \mathrm{min}$ ) and $U_{\text {ave }}^{\max } D=1.47 \mathrm{~mm}^{2} / \mathrm{s}$ of our pump are useful for microelectronics cooling with a small package size. Further, although the drive voltage is relatively high, we can 
also improve it by reducing $W$ in the future, e.g., by the rough scaling argument, i.e., by reducing the size of $W 1 / 33(\simeq 1.06 / 35)$ times, we can obtain $U_{\text {ave }} \sim 0.94 \mathrm{~mm} / \mathrm{s}$ under the condition that $V_{0}=1.06 \mathrm{~V}$ and $W=45.4 \mu \mathrm{m}$, since $U_{\text {ave }}$ is proportional to the square of the electric field $E=V_{0} / W$. In other words, the ability to convey a liquid of our ratchet ACEO pump is approximately $1.3(=45.4 \times 0.94 / 25 \times 1.3)$ times larger than that of the $3 \mathrm{D}$ array-type ACEO pump at $V_{0}=1.06 \mathrm{~V}$. In detail, we need to consider the increase of $R_{c}$. Specifically, from the relation of $R_{c}=\frac{12 \mu}{W^{3}} \frac{L}{D}, R_{c}$ and $U_{\text {ave }}$ may increase by $33^{3}=35937$ and $1 / 35937$ times, respectively, at $W=45.4 \mu \mathrm{m}$, if the surface of the channel wall works as a sticking adhesion boundary. However, since the surface of the ratchet electrode at least works as a slip boundary, this argument is not acceptable. Further, the problem will be overcome easily by using a two-ratchet electrode structure in the future. Thus, we consider that our device is useful, although it is important to prove the performance of the small gap device in the future.

\section{E. Microfluidic Rectifier Effect}

The Reynolds number $R e(=U W / \nu)$ of the system is 2.1 at most, where $U\left(\simeq U_{\max } \simeq 1.4\right.$ $\mathrm{mm} / \mathrm{s})$ represents velocity and $\nu\left(\simeq 10^{-6} \mathrm{~m}^{2} / \mathrm{s}\right)$ is the kinematic viscosity for water. Thus, the fluidic ratchet effect, which is known for the Newtonian fluid at high $R e^{12}$, might not be expected in the present system. However, if we can improve $U_{\max }$ (or reduce $\nu$ ), the effect may be preferable. In particular, Groisman and Quake ${ }^{11}$ already reported that a microfluidic channel having a ratchet structure has a characteristic of a microfluidic rectifier for the non-Newtonian fluid such as a $0.01 \%$ aqueous solution of a high-molecular-weight polymer. Thus, there is a possibility that our ratchet ACEO pump shows higher pumping performance for non-Newtonian fluids in the future because of the global asymmetrical structure of the device, although we will need to examine it in the future. Moreover, since $R e \sim 2$, the turbulent vortices do not affect the present experiment, although we mentioned the vortices due to the ratchet in Sec. III. 


\section{F. Optimization of the Ratchet Sizes}

Since the electro-osmotic slip velocity $V_{s}$ is proportional to $\left(V_{0} / W\right)^{2}$, a large $d_{2}$ results in low performance. Specifically, if the slip velocity at the slope position $s$ is assumed as $V_{s 0} /(1+s \theta / W)^{2}$, the average slip velocity over $d_{2}$ is obtained as $V_{s}^{\text {ave }}\left(d_{2}\right)=$ $\frac{1}{d_{2}} \int_{s=0}^{s=d_{2}} \frac{V_{s 0}}{\left(1+\frac{\theta s}{W}\right)^{2}} d s=\frac{V_{s 0}}{\frac{\theta d_{2}}{W}+1}$, where $V_{s 0}$ is the slip velocity at $s=0$ (i.e., at the peak of the ratchet), $\theta=\tan ^{-1}\left(d_{1} / d_{2}\right) \simeq 0.25$, and $s$ is the distance from the ratchet peak. Therefore, mathematically, $V_{s}^{\text {ave }}\left(d_{2}\right)$ approaches $V_{s 0}$ in the limit $\frac{\theta d_{2}}{W} \rightarrow 0$ and the condition provides maximum performance. However, in a real design, we require $d_{1}, d_{2} \gg \delta$ to obtain the ratchet structure, where $\delta$ is the surface roughness. Further, we obtain $V_{s}^{\text {ave }}\left(d_{2} \theta / W\right)=V_{s 0} / 1.2 \simeq 0.83 V_{s 0}$ at $d_{2} \theta / W \simeq d_{1} / W=0.3 / 1.5=0.2$. Thus, our ratchet does not deteriorate the performance too much compared to the ideal ratchet. In other words, the condition $\delta \ll d_{1} \ll W$ is recommended to maintain a good performance for a small value of $\theta$.

\section{G. Physical Picture Due to a Motion of a Vorticity}

As we mentioned before that the size of the vortex becomes large as the vortex flows disappear, we here explain it in a more sophisticated manner by considering the motion of a vorticity $\omega \boldsymbol{k}=\nabla \times \boldsymbol{u}$, where $\boldsymbol{u}$ is the flow velocity vector and $\boldsymbol{k}$ is a unit vector in the $z$ direction. In particular, by using the concept of vorticity dynamics ${ }^{19}$, we explain the phenomenon. In the initial stage, since water is stationary, large vorticities are generated at each slope of the ratchet electrode due to the electro-osmotic slip velocity. Gradually, these vorticities are conveyed by the flow and diffused according to the vorticity equation, i.e., $\frac{\partial \omega}{\partial t}+\boldsymbol{u} \cdot \nabla \omega=\nu \nabla^{2} \omega$. Further, The flow fields at the position $\boldsymbol{x}$ and time $t$ are calculated by the Biot-Savart law, i.e., $\boldsymbol{u}(\boldsymbol{x}, t)=-\frac{1}{2 \pi} \iint \frac{\left(\boldsymbol{x}-\boldsymbol{x}^{\prime}\right) \times \omega \boldsymbol{k}}{\left|\boldsymbol{x}-\boldsymbol{x}^{\prime}\right|^{2}} d S\left(\boldsymbol{x}^{\prime}\right)$. Here, the sizes of the vortices are approximately defined by the size of the vortex patch (or vorticity distribution region), which is considered to be uniform or to be a unit of periodic distribution. In our transient response problem, the vortex layer of the size $d_{2}$ grows into a vortex patch of the size $d_{2} \times W$ in the early stage. Then, by the diffusion due to the viscosity, the vorticity distribution becomes smooth in the bulk region of the channel, whereas the vortex patch near the inlet expands by the transportation of the vorticity due to the flow. Thus, the 
smoothing process of the vorticity distribution proceeds from the inlet to the bulk region of the channel. Further, if $U_{\text {ave }}$ approaches the electro-osmotic slip velocity of the ratchet electrode in the final stage, the generation of the vorticity at the surface of the ratchet electrode is suppressed.

\section{CONCLUSION}

In conclusion, we proposed a ratchet ACEO pump that uses a ratchet electrode faced to the plane electrode and demonstrated its high net flow of $\sim 1 \mathrm{~mm} / \mathrm{s}$. Moreover, we directly showed that it does not produce any vortex flow in the channel when it produces a high speed net flow. The ratchet ACEO pump is useful since it produces a large net flow without a complicated optimum design. Furthermore, the performance of our pump can also be improved for non-Newtonian fluids and high-Reynolds-number systems.

\section{ACKNOWLEDGMENTS}

This work was partially supported by JSPS KAKENHI Grant Number JP16K05650.

\section{REFERENCES}

${ }^{1}$ T. Squires and S. Quake, "Microfluidics: Fluid physics at the nanoliter scale," Rev.Mod.Phys. 77, 977 (2005).

${ }^{2}$ Y.-N. Wang and L.-M. Fu, "Micropumps and biomedical applications a review," Microelectronic Engineering 195, 121 - 138 (2018).

${ }^{3}$ A. Ajdari, "Pumping liquids using asymmetric electrode arrays," Phys. Rev. E 61, R45 (2000).

${ }^{4}$ A. Ramos, A. Gonzalez, A. Castellanos, N. G. Green, and H. Morgan, "Pumping of liquids with ac voltages applied to asymmetric pairs of microelectrodes," Phys. Rev. E 67, 056302 (2003).

${ }^{5}$ J. P. Urbanski, T. Thorsen, J. A. Levian, and M. Z. Bazant, "Fast ac electro-osmotic micropumps with nonplanar electrodes," Appl. Phys. Lett. 89, 143508 (2006). 
${ }^{6}$ S. Yao, A. M. Myers, J. D. Posner, K. A. Rose, and J. G. Santiago, "Electroosmotic pumps and their applications in microfluidic systems," J. Microelectromech. Syst. 15, 717 (2006).

${ }^{7}$ A. Ramos, H. Morgan, N. G. Green, and A. Castellanos, "Ac electric-field-induced fluid flow in microelectrodes," J. Colloid Interf. Sci. 217, 420 (1999).

${ }^{8}$ M. Z. Bazant and T. M. Squires, "Induced-charge electrokinetic phenomena: Theory and microfluidic applications," Phys. Rev. Lett. 92, 066101 (2004).

${ }^{9}$ A. Brown, C. Smith, and A. Rennie, "Pumping of water with ac electric fields applied to asymmetric pairs of microelectrodes," Phys. Rev. E 63, 016305 (2000).

${ }^{10} \mathrm{M}$. Bazant and Y. Ben, "Theoretical prediction of fast 3d ac electro-osmotic pumps," Lab on a Chip 6, 1455 (2006).

${ }^{11}$ A. Groisman and S. R. Quake, "A microfluidic rectifier: Anisotropic flow resistance at low reynolds numbers," Phys. Rev. Lett. 92, 094501 (2004).

${ }^{12}$ H. Linke, B. J. Alemán, L. D. Melling, M. J. Taormina, M. J. Francis, C. C. Dow-Hygelund, V. Narayanan, R. P. Taylor, and A. Stout, "Self-propelled leidenfrost droplets," Phys. Rev. Lett. 96, 154502 (2006).

${ }^{13}$ J. P. Urbanski, J. A. Levitan, D. N. Burch, T. Thorsen, and M. Z. Bazant, "The effect of step height on the performance of three-dimensional ac electro-osmotic microfluidic pumps," Journal of Colloid and Interface Science 309, 332 - 341 (2007).

${ }^{14}$ N. V. Deshpande, "Significance of inertance and resistance in fluidics of thermal ink-jet transducers," Journal of Imaging Science and Technology 40, 396 (1996).

${ }^{15}$ T. Bourouina and J.-P. Grandchamp, "Modeling micropumps with electrical equivalent networks," Journal of Micromechanics and Microengineering 6, 398 (1996).

${ }^{16}$ A. Asai, T. Hara, and I. Endo, "One-dimensional model of bubble growth and liquid flow in bubble jet printers," Japanese Journal of Applied Physics 26, 1794 (1987).

${ }^{17}$ D. J. Laser and J. G. Santiago, "A review of micropumps," Journal of Micromechanics and Microengineering 14, R35 (2004).

${ }^{18}$ C.-C. Huang, M. Bazant, and T. Thorsen, "Ultrafast high-pressure ac electro-osmotic pumps for portable biomedical microfluidics," Lab on a Chip 10, 80 (2010).

${ }^{19}$ A. Leonard, "Vortex methods for flow simulation," Journal of Computational Physics 37, 289 (1980). 